\title{
Home Appliances Monitoring System using a Hall Element
}

\author{
Hiromasa Tomimoto $^{\mathrm{a},}$, Yoshikazu Hitaka ${ }^{\mathrm{a}}$, Shota Nakashima ${ }^{\mathrm{b}}$, Kazuo Haruyama $^{\mathrm{a}}$ \\ ${ }^{a}$ Ube National College of Technology, 2-14-1 Tokiwadai, Ube, Yamaguchi 755-8555, Japan \\ ${ }^{\mathrm{b}}$ Graduate School of Science and Engineering, Yamaguchi University, 2-16-1 Tokiwadai, Ube, \\ Yamaguchi 755-8611, Japan \\ *Corresponding Author: haruyama@ube-k.ac.jp
}

\begin{abstract}
In recent years, Japanese has been faced with an increasingly aging society with a decreasing birth rate, which has resulted in problems such as a lack of care workers and an increasing number of elderly people living alone. In Japan, an increase in the aging society is predicted, thereby worsening the abovementioned problems. Therefore, it will become more difficult for elderly people living alone to receive care. We propose a Home Appliances Monitoring System to confirm their safety. This system employs a Hall element for detecting elderly persons when using home appliances. When a home appliance is used, an electric current passes through its power cord, and a magnetic flux is produced around the power cord. The Hall element converts the magnetic flux into a voltage. Based on the voltage, the system determines whether the home appliance was used. The proposed method can be applied to all home appliances, except specific cord.
\end{abstract}

Keywords: elderly people, home appliances, Hall element, magnetic flux.

\section{Introduction}

In October 2012, Japan's total population was estimated to be 127.8 million, of which the number of elderly persons (aged 65 or over) was estimated to be about 30.8 million. This figure accounts for $24.1 \%$ of the total population is the highest on record. In addition, the Statistics Bureau has predicted that this percentage will be $39.9 \%$ in $2060^{(1-3)}$. Currently, the increasingly aging society, together with a decreasing birth rate, is leading to problems such as the lack of care workers and growing number of elderly persons living alone and elderly households. These problems are expected to become more serious, thereby making it difficult for elderly people living alone to receive care. As a result, the elderly would not be detected if confronted by accidents. Also, they could sometimes encounter multiple accidents, leading to a lonely death. To address these problems, methods have been proposed to monitor the elderly using surveillance cameras installed at their homes ${ }^{(4,5)}$. In this way, observers can swiftly respond to problems that may arise. However, elderly person may be repelled by the idea of being monitored by surveillance cameras. Also, monitoring is undesirable from the privacy aspect. Therefore, recently, there have been several studies focusing on verifying the safety of elderly persons without the use of surveillance cameras ${ }^{(6-9)}$. On the other hand, some companies have already developed and marketed systems to realize this goal. They provide services that notify relatives living in distant places of the occurrence of any accidents. However, the relative required to purchase specialized devices. In this paper, we propose a system that is more widely applicable.

\section{System}

The system consists of a detector, power line communication (PLC) and information processing communication (IPC). Fig.1 shows a diagram of our proposed Home Appliances Monitoring System. First, the detection part (Hall element) is attached to the power cord of a home appliance. Second, the Hall element output voltage is used as the signal when the home appliance is used. After inputting the signal to the peripheral interface controller (PIC), the PIC determines whether the home appliance was used by measuring magnitude of the signal. Third, the detector transmits a detection data to the IPC through the PLC. Finally, the IPC sends monitoring information using the packet radio terminal to the server, which is used to inform the relatives of the elderly person. From this information, the relatives can know that the elderly person is safely if the home appliances are used in a 


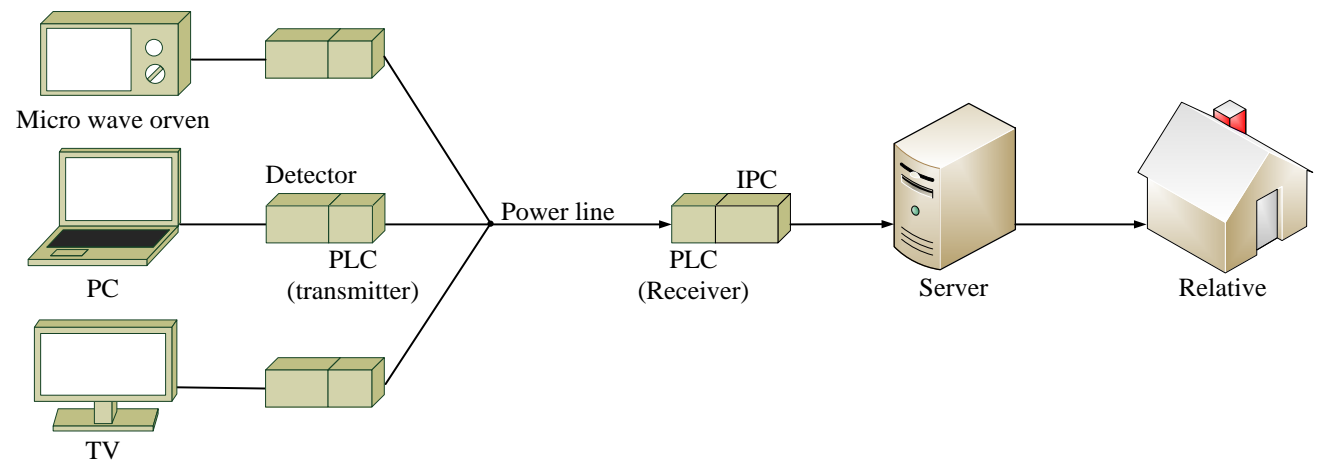

Fig.1. Home Appliances Monitoring System

normal manner. The relatives can also know that the elderly person has experienced some problem when home appliances are not used for a long time or if they are used at an unscheduled time. They would then be able to initiate attempts to contact and assist the elderly person.

\subsection{Hall element}

This system uses a Hall element to the use of home appliances by the elderly. Fig.2 shows the structural drawing of the Hall element. The Hall element is made from semiconductors, and if an electric current is passed through it and magnetic flux enters it perpendicularly, the Hall element generates a voltage that is perpendicular to both. This voltage is called the Hall voltage $V_{H}$, and its value depends on the Hall coefficient $K_{H}$, the current $I$, and the magnetic flux density $B . V_{H}$ is calculated according to the following equation.

$$
V_{H}=K_{H} I B
$$

Here $K_{H}$ is considered to be a constant. $V_{H}$ varies with changes in only $B$ by smoothing $I$ using a constant current circuit. In other words, the Hall element converts the magnetic flux into a voltage, which is used in the assessment.

\subsection{Detector}

The detector consists of a detection circuit (Hall element, amplifier, and frequency filter) and judgment. Fig.3 shows internal structure of the detector. From equation (1), we see that the Hall voltage is proportional to the magnetic flux density. However, the Hall voltage decreases, because the actual magnetic flux density is very small. Thus, two amplifiers with operational amplifiers amplify the Hall voltage. Next, the band-pass filter (BPF)

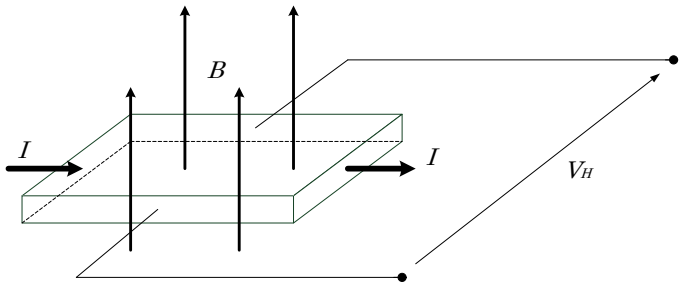

Fig.2. Structural drawing of the Hall element

cleans the noise to increase the selectivity. Specifically, it limits the frequency band to $60 \mathrm{~Hz}$ because the frequency of the Hall voltage is $60 \mathrm{~Hz}$. Finally, the low-pass filter (LPF) cleans noise to a greater extent, because there is a high-frequency component. The total gain is twenty thousand. The output of this filter is read into the PIC, and it determines whether the home appliance was used.

\subsection{Assessment method}

The output waveform of the detection circuit varies with the type of home appliance. Thus, the judgment calculates an area of the waveform and judges the detection from the area. Fig.4 shows the flowchart of program written into PIC for detection. The detection occurs as described below.

First, the detector reads the output voltage of the detection circuit $N$ times when the system is powered on, and averages the read voltages. It then calculates the area of the waveform on the basis of this average. Next, the detector reads the instantaneous value of the output voltage, and calculates the difference between the read value and the instantaneous value. It then repeats the job 320 times per cycle. The detector treats the sum of the difference as the area. The count is then incremented by one if the area is more than the sat area. The maximum value of the count is

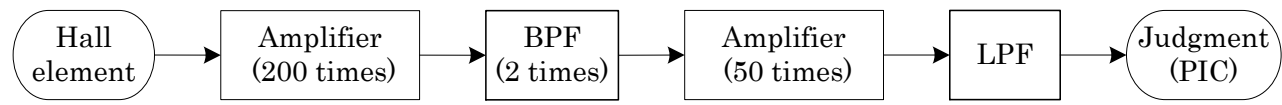

Fig.3. Internal structure of the detector 
60 times, since the frequency of the output signal is identical to the commercially available frequency. Thus, we determine that the judgment can detect operation of the home appliance if the count exceeds 95\% (57 times) or more of the maximum value. The detector sends the detected data to the IPC through the PLC when the count exceeds 57 times a second.

\section{$2.4 \quad$ PLC}

The PLC communicates from the detector to the IPC using a power line as a communication pathway. This can be easily implemented, because there is no need for additional communication wires to be run. The system has a low-speed PLC, which has a low transmission rate. The PLC communicates with a frequency band of $450 \mathrm{~Hz}$ or less and has a marginal effect on radio communication or medical equipment because of its low frequency. In addition, it is characterized by high reliability, because the signal-to-noise ratio is superior. It is necessary to send definite information, because the proposed system aims to confirm the elderly person's wellbeing to their relatives. Also, a high-speed PLC is not necessary, because the system does not deal with a large amount of data. Therefore, we chose a low-speed PLC.

\section{Results}

\subsection{Way of experiment}

We examined the usefulness of the system by performing experiments using home appliances based on the following procedure. (1) The home appliance and the system's transmitter are plugged into the outlet. Also, the system's receiver is plugged into other outlet in same room.

(2) The Hall element is attached to the power cord of the home appliance. (3) The system is turned on. After that, we wait for about 2 seconds to calculate the reference voltage at the detector. (4) The home appliance is turned on. We confirm that the system's receiver lights up. It turns out that the system can detect when the system's receiver is lit. (5) The Hall element is detached from the power cord. The magnetic flux density is measured at the point touching the Hall element. We experimented with each home appliance using the above method.

Other confirmation system has a good track record for communicating between the PLC and the IPC ${ }^{(10)}$. Thus, we omit the IPC and experiment.

\subsection{Experimental results}

We experimented with 13 different home appliances.

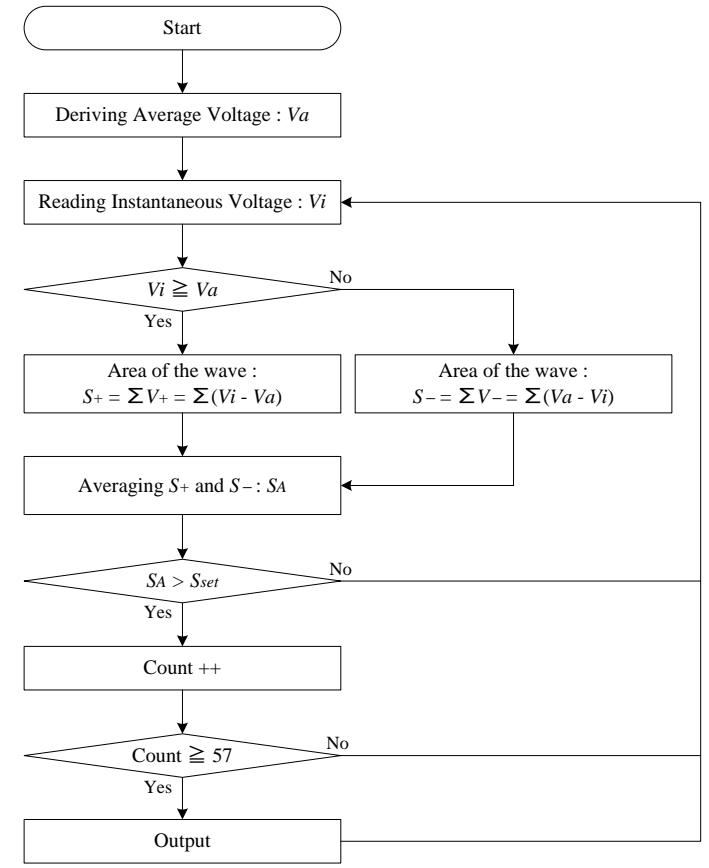

Fig.4. Flowchart for detection

The system detected the operation of 10 home appliances. In addition, there were no false-positive scenarios, where the appliance was determined to be on when it was in fact off. However, the system could not detect the operation of three home appliances despite performing many experiments. Fig.5 shows the variation of magnetic flux density with power. As seen in the figure, the magnetic flux density is proportional to power for the detectable home appliances. However, the magnetic flux density is very small, regardless of the power for the undetectable home appliances.

\subsection{Analysis}

There was only a small magnetic flux around the cord of undetectable home appliances. Hence, the detector could not detect their operation. We analyzed the effect of each power cord, and it was found that the magnitude of the magnetic flux density is related to the type of cord. All undetectable home appliances had the same type of power cord, i.e., vinyl cabtyre flat (VCTF) cable.

Fig.6 shows the power vs. magnetic flux density chart for VCTF and non-VCTF cables. It is apparent that the magnetic flux density around VCTF cables is very small compared to that around other cords. Fig.7 shows the structure of a VCTF cable, where two lines are twisted and covered with thicker vinyl. Therefore, a VCTF cable would leak only a small magnetic flux to the outside. Therefore changes in the magnetic flux are undetectable by the Hall element. 


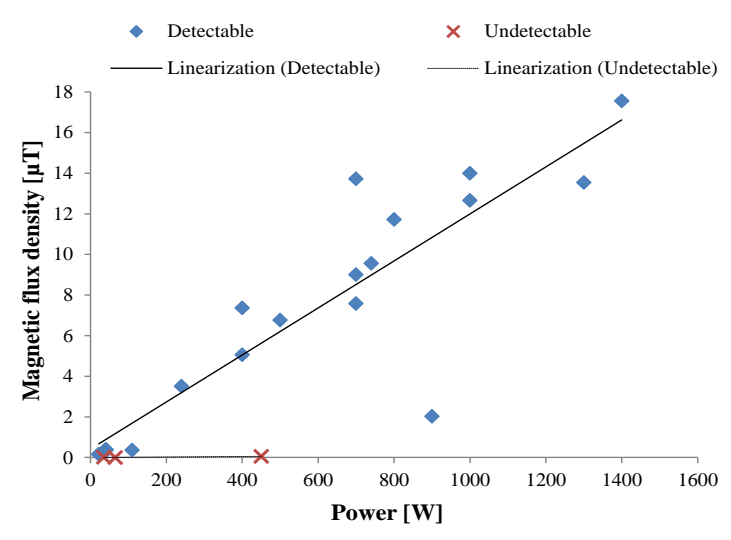

Fig.5. Power-Magnetic flux density

(Detectable and Undetectable)

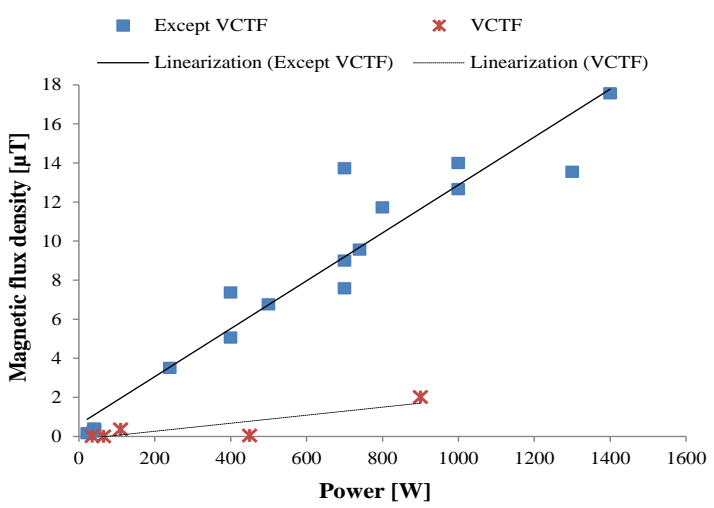

Fig.6. Power-Magnetic flux density (VCTF and Except VCTF)

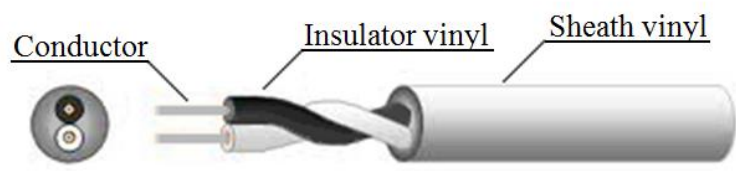

Fig.7. Structure of VCTF cable

\section{Conclusions}

We developed a system that performs the primary task of sensing the operation of several appliances. However, the system is currently unable to detect the operation of home appliances with VCTF cables because the magnetic flux density is very small. However, the system did not determine that home appliances were being used when they were not actually being used. Also, the proposed method can be applied to all home appliances, with the exception of those having VCTF cables. Therefore, the system is considered to be functional.
The future challenge is to investigate the effect of all type of power cords on the magnetic flux density. If there are other cords that have no effect on the magnetic flux density, we will conduct field tests as necessary.

\section{Acknowledgment}

This work was supported by JSPS KAKENHI 24500670

\section{References}

(1) Statistics Bureau, Ministry of Internal Affairs and Communications: "Population Estimates" (2013)

(2) Ministry of Health, Labour and Welfare: "Changes in the enrollment of care worker" (2013)

(3) Cabinet Office, Government of Japan: "White Paper on Aging Society 2013" (2013)

(4) M. Doi, H. Inoue, Y. Aoki and O. Oshiro: "Video Surveillance System for Elderly Person Living Alone by Person Tracking and Fall Detection," The Institute of Electrical Engineers of Japan. E, Vol.126, No.8, pp.457-463 (2006)

(5) H. Seki and Y. Hori: "Detection of Abnormal Action Using Image Sequence for Monitoring System of Aged People," The Institute of Electrical Engineers of Japan. D, Vol.122, No.2, pp.182-188 (2002)

(6) K. Tanaka, K. Haruyama and Y. Yamada: "Safety Confirmation System Using Mat-Sensor and Power Line Communications for Elderly Person," Journal of Robotics and Mechatronics, Vol.19, No.6, pp.676-682 (2007)

(7) K. Ota, M. Otsu, Y. Ota and A. Kajiwara: "Elderly-Care Motion Monitoring Sensor Using Ultra-wideband Radio," The Institute of Electrical Engineers of Japan. C, Vol.131, No.9, pp.1547-1552 (2011)

(8) S. Kondo: "Safety Confirmation System for Elderly Single-person Household with Sensor Systems," The Institute of Electrical Engineers of Japan. C, Vol.131, No.7, pp.1293-1297 (2011)

(9) T. Oyabu, S. Hirobayashi, H. Kimura: "Monitoring System for a House of the Solitary Elderly Person using Multiple Tin-oxide Gas Sensor," The Institute of Electrical Engineers of Japan. A, Vol.117, No.6, pp.314-320 (1997)

(10) K. Tanaka, A. Uchibori, K. Haruyama, Y. Nishimura, S. Uchikado, Rahman Faridah Abd: "Safety Confirmation System Using Ultrasonic Radar for Elderly People Living Alone," The Institute of Electrical Engineers of Japan. D, Vol.131, No.2, pp.202-207 (2011) 